\title{
Human and Environmental Analysis of Wearable Thermal Energy Harvesting
}

\author{
Amanda Myers ${ }^{a}$, Ryan Hodges ${ }^{b}$, Jesse S. Jur ${ }^{c}$
}

\section{Department Affiliations}

a. Department of Mechanical and Aerospace Engineering, North Carolina State University, Raleigh, NC

b. Department of Electrical Engineering, North Carolina State University, Raleigh, NC

c. Department of Textile Engineering, Chemistry and Science, North Carolina State University, Raleigh, NC

* Corresponding Author Contact Information: Dr. Jesse S. Jur, jsjur@ncsu.edu, 2401 Research Drive, Campus Box 8301, Raleigh, NC 27695-8301, Phone: (919) 515-1676, Fax: (919) 515-6532

\section{Abstract}

In considering wearable energy harvesting, one must recognize the wide array of factors that lead to variations of energy harvesting. The objective of this work is to define analytical methods to study the effect of environmental and human factors on thermal energy generator (TEG) performance in a variety of use case scenarios. A test method for evaluating the performance of a TEG in a wearable form is developed and demonstrated using both in-lab and out-of-lab procedures. The fabrication procedure of an energy harvesting wearable device demonstrates a method of integrating rigid devices into a flexible substrate. The wearable device is used in a human trial which covered a series of activities in different environmental conditions. The results of these trials demonstrate the significant effect of movement, or convection, on thermal energy harvesting. Humidity levels do not have a significant correlation to power; however, wet bulb temperature must be taken into consideration due to the additional cooling effect of evaporation on temperature. The data collected indicates that while dry-bulb temperature does not have the greatest effect on TEG power generation, wet-bulb temperature is indicative of TEG performance. Additionally, user generated movement is the main consideration when designing a wearable device with TEGs as it had the largest effects on power generation. The results of this work 
1 quantify how a wearable device will perform throughout daily activities, allowing the definition of an

2 operational scenario of a self-powered wearable device while choosing the most appropriate design for

3 a particular application. This work also provides a foundation for exploring how textiles can enable the

4 design of unique wearable devices. This will lead to further investigation into quantifying the effect that

5 the construction of a textile has on TEG performance as well as on consumer comfort.

\section{$6 \quad$ Keywords}

7 Wearable Technology; Energy Harvesting; Thermoelectric Devices; Environmental to Human

8 Correlations

\section{Introduction}

Recent advances in low power electronics, including semiconductor on chip and radios are enabling 11 opportunities in wearable devices that utilize sources of energy outside the use of a battery. In 12 wearable devices, batteries add both bulk and weight to a device reducing its perceived comfort [1-4], 13 and also require plug-in recharging that can seem burdening to the consumer. While low power 14 electronics enable strategies in reducing the battery size or improving the operating lifetime between 15 battery charges, the ability to use energy harvesting directly from the body has been suggested as an 16 alternate source of power. Studies exploring mechanical and thermal energy harvesting have shown 17 promise in guiding energy harvesting materials design, but have been limited to benchtop and short term use case studies [5-8]. The energy harvesting packaging design for on-body use has a number of complex design criteria that define the device comfort and performance. For example, the on-body 20 location of the device identifies form factor, flexibility, materials used, etc. that need to be considered 21 with regard to user comfort. The device performance is highly dependent on human factors that are

22 even more influential to the performance of the energy harvester [9-11]. For a thermal energy 23 generator (TEG), human factors include the temperature difference between the surface of the skin and 
1 ambient air, air velocity over the TEG, and the humidity of the ambient air, resulting in a complex

2 combinatorial effect on the energy harvesting ability of the TEG. The influence of these variables is

3 dependent on the harvester's location on the body. This work aims to explore testing strategies for

4 determining the effect of human factors related to thermal energy harvesting, including environmental

5 influences and position, or location, of energy harvesting, which thereby enables future strategic design

6 of the materials surrounding the energy harvesting for improving performance.

8 Prior research has shown that thermal energy harvesting offers a consistent supply of energy for wearable energy harvesting purposes[12-16]. Of particular note is the work conducted by Leonov et al. $[12,15,17,18]$ that have demonstrated TEG performance in a watch-based and shirt based platforms.

11 Their research demonstrated that the key challenge of thermal energy harvesting from the body is the 12 temperature differential between the body and microclimate surrounding the device that ultimately 13 limits the energy that can be harvested. The common approach to improve thermal energy harvesting is 14 to include a heat spreader and/or a heat sink to improve power output and maintain a temperature 15 gradient across wearable TEGs. The rigid and bulkiness of the heat sink comes at the expense the 16 perceived comfort of the device. Settaluri et al. [19] characterized and optimized a heat sink and heat 17 spreader system for use with a wearable TEG wristband, acquiring a power density of $28.5 \mu \mathrm{W} / \mathrm{cm}^{2}$. 18 Another is the fabrication of flexible or fiber-based TEGs [8,20-22] which improves the TEG-to-skin contact. Both of these methods highlight the importance of improving TEG performance on a device 20 level in a more comfortable manner, but do not consider the variety of environmental conditions the 21 human body is exposed to on a daily basis. Of particular interest is the observation that thermal energy 22 harvesting has been shown to have negative effects on human comfort due to a high heat flow from the 23 skin resulting in a chilling effect $[17,23]$. For the eventual success of on-body energy harvesting, the 24 ability to understand what factors influence energy harvesting over the wide range of body placement 
1 and human factors is paramount to mitigating human discomfort without compromising the efficiency

2 of the thermal energy harvester.

4 The purpose of this work is to quantify the effects of transient environmental conditions and human

5 scenarios on the efficiency of a body-worn TEG via controlled laboratory experiments and human trial

6 studies. The controlled tests presented in this work characterize the TEG with defined heat loads, air

7 velocities, and heat sinks to define TEG integration strategies. Using these design strategies, a wearable

8 platform is used to collect experimental data directly from a human in true indoor and outdoor

9 conditions. As part of the device design, a novel printed circuit board (PCB) design was constructed to

10 simultaneously monitor the environmental conditions, activity level of the human, and the

11 instantaneous power generated by the TEG device. Data was collected using this platform in real time

12 with human subjects performing different activities that changed motion and environmental conditions.

13 The results of this work identify specific environmental variables that show the largest effect on the 14 energy harvested and analysis is performed to define correlations between motion intensity and power 15 generation. Finally, a power map based on activity and external environment is defined that can be 16 used to estimate TEG efficiency in future studies.

\section{Methods for TEG Characterization and Human Trials} characterize the response of the TEGs (Laird Technologies: OT08, 18, F0, 0505, 11) used in this study. In a benchtop analysis, finned heat sinks with values of $10{ }^{\circ} \mathrm{C} / \mathrm{W}, 4.6^{\circ} \mathrm{C} / \mathrm{W}$, and $1.9^{\circ} \mathrm{C} / \mathrm{W}$ (CTS Electronics

21 Components) were each applied to the TEG with matched load (4.4 $\Omega$ ) and the power output recorded.

22 The TEG was placed in contact with a thin layer of polydimethylsiloxane (PDMS) situated in contact with

23 a hot plate. The PDMS served as a thermal simulant to skin (the thermal conductivity of PDMS is 0.15 
$1 \mathrm{~W} / \mathrm{mK}[24]$ while the thermal conductivity of the epidermis layer of skin ranges from $0.2-0.5 \mathrm{~W} / \mathrm{mK}$

2 [25]) and the PDMS was able to dampen the effects of the temperature fluctuations of the hot plate 3 with minor spatial variability. A hot plate temperature of $37{ }^{\circ} \mathrm{C}$ resulted in a PDMS exposed surface

4 temperature of $35{ }^{\circ} \mathrm{C}$, which is an average temperature of human skin.[26] During all experiments, the

5 ambient air temperature remained between $21-22{ }^{\circ} \mathrm{C}$. After the TEG power output reached steady state

6 in stagnant air ( $30 \mathrm{~min})$, an air flow of $1.2 \mathrm{~m} / \mathrm{s}$ was provided using a small fan placed $15 \mathrm{~cm}$ away from

7 the heat sink edge to simulate the airflow induced by a human walking pace [27]. This setup is similar to 8 that demonstrated previously in testing for a material's Seebeck coefficient for a TEG placed on the body 9 [28].

10 The effect of spacing between TEGs was evaluated by placing two TEGs in series at increasing spaces.

11 The spacing between the TEGs ranged from $0 \mathrm{~mm}$ to $6 \mathrm{~mm}$ as measured between the neighboring edge

12 of each device, and held in place with in a structural mold. The TEGs were placed on the heated PDMS

13 with a matched resistive load $(8.8 \Omega)$ and the experiment outlined previously was repeated.

142.2 On-Body TEG Evaluation. The construction of the wearable TEG devices is outlined below in terms of

15 the formation of the flexible TEG integration into a textile assembly and the construction of a novel 16 evaluation platform for communicating the data wirelessly from the body to an Android tablet. Finally, 17 the experimental design for the human trial study is described.

18 The design for integrating a series of TEGs into a flexible package is outlined in Fig. 1a. Pyralux ${ }^{\circledR}$ 19 (Dupont), a flexible Kapton substrate that is coated in $\mathrm{Cu}$ on one side, serves as the flexible substrate 20 which connects the TEGs. Pyralux ${ }^{\circledR}$ substrate is prepared using a die cutter to create five holes, $4.9 \mathrm{~mm} \mathrm{x}$ $214.9 \mathrm{~mm}$, spaced $6 \mathrm{~mm}$ apart horizontally as dictated by the controlled lab experiments presented. A 22 mask applied to the Pyralux ${ }^{\circledR}$ defines the $\mathrm{Cu}$ connections between TEGs. The exposed $\mathrm{Cu}$ is etched by 23 submersing the substrate in ferric chloride for $>15 \mathrm{~min}$. The mask is removed to expose the copper 
1 interconnects. The TEG is integrated into the cut out holes by first placing a Bi-Sn low temperature

2 solder on the $\mathrm{Cu}$ coated sides to the gap that the TEG are placed, with the phalanges of each TEG

3 maintaining direct contact with the solder. The substrate with the TEGs is cured in a reflow oven for 420

$4 \mathrm{~S}$ at a maximum temperature of $150^{\circ} \mathrm{C}$.

52.3 Device Assembly. The flexible substrate with the TEGs integrated is embedded in a textile

6 multilayered structure comprising of Cu-coated taffeta, a polyethylene nonwoven textile, and jersey

7 polyester knit. The Cu taffeta acts as a heat spreader between the skin and lower header of the TEGs.

8 The polyethylene is included as structural support of the TEGs and as an insulating layer between the

9 top and bottom of the TEGs. The polyester knit serves as the base fabric for the wearable device. The

10 top edges of the band are sewn together and a $30 \mathrm{~mm} \times 125 \mathrm{~mm}$ area is cut from the knit and Cu taffeta

11 is sewn in its place. Small holes are cut in the outer polyester layer and in the inner polyethylene layer

12 for TEG insertion. A fastener is attached to the band to accommodate size variations between human

13 subjects. The TEG series attaches to the band using alumina thermal paste on the TEG/Cu taffeta

14 interface. Aluminum heat sinks, exposed to the environment, adhere to each TEG header with carbon

15 tape. The final profile of the assembly is demonstrated in Fig. 1c. The data collection board, EHSS, is

16 mounted to each of the devices via a mesh pocket. The pocket provides mechanical protection for the

17 electronics without inhibiting any sensing modalities. The resulting devices as worn by a mannequin are

18 displayed in Fig. 1b. 

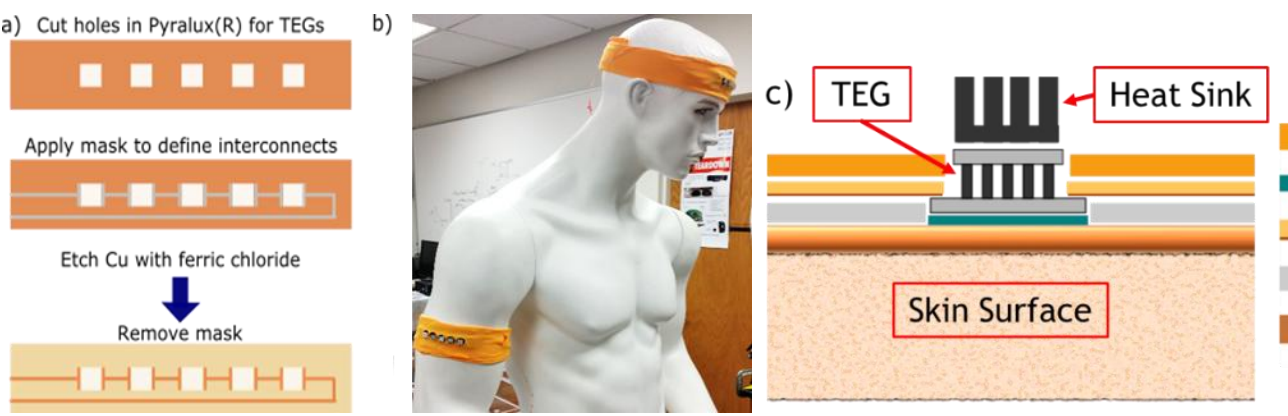

Polyester Knit

Alumina Thermal Paste

Pyralux Substrate

Polyethelyne Fabric

Thermally Conductive Copper Fabric

Figure 1: a) Overview of flexible circuit fabrication process b) Fabricated headband and armband c)

2.4 EHSS Platform Design. A unique energy harvesting platform was developed that allows for simultaneous energy harvesting and storage evaluation alongside incorporation of sensors. The batterypowered platform and corresponding source code is based on the TI SensorTag development kit. The evaluation system is specifically designed to measure the useable current and voltage from an energy harvester the power consumption from a suite of sensors. Moreover, the PCB dimensions and communication allow for simple on-body testing. The system's primary differentiation to commercial systems is its application as a testing platform for real time power management profiles necessary for development of on-body self-powered sensor systems.

12 The energy harvesting subsystem (EHSS) allows for the wireless transmission of multiple sensor and energy harvesting data via Bluetooth LE. It measures harvested voltage and current levels from thermoelectric, piezoelectric, and/or solar energy harvesting devices delivered to a variable load. In addition, the system measures 3-axis accelerometer data, 3-axis gyroscope data, ambient temperature, and humidity. A block diagram for the system is seen in Fig. 2. Output from the TEG is first sent to a

17 transformer and boost converter (LTC3108) [29] before getting sent to measurement and load circuits.

18 Other sensor data communicates directly with the microcontroller using an I2C interface. The firmware for the on-board microcontroller has been developed to wirelessly transmit all sensor data and is 
1 designed for future expansion should additional sensing modalities be required. The accelerometer data

2 is transmitted to an app on a Bluetooth-enabled device at a rate of $100 \mathrm{~Hz}$ while all other data rates are

3 set to $1 \mathrm{~Hz}$. Additionally, the app logged each data point to allow for real time plotting and for the data

4 to be accessed at a later point.
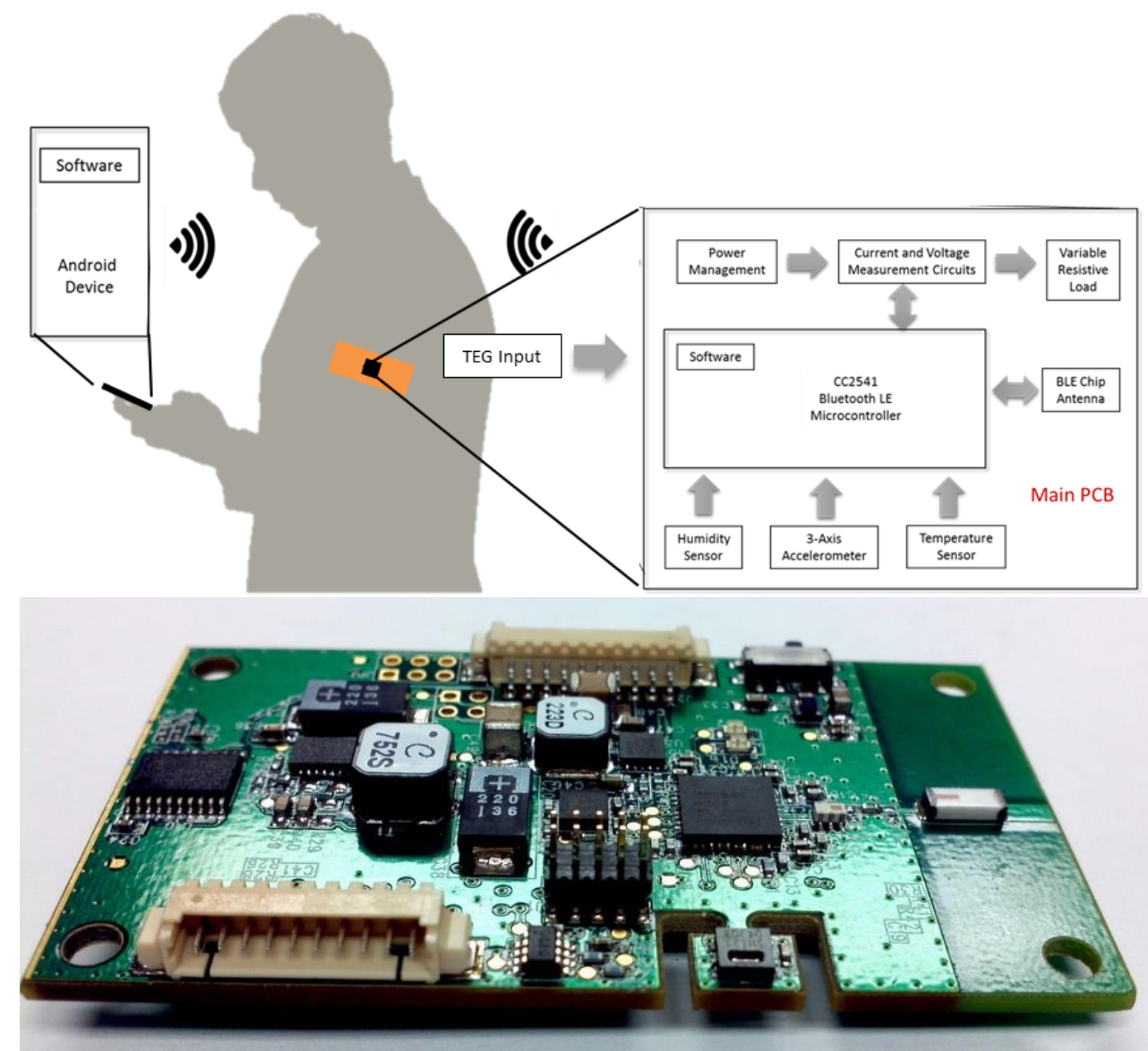

Figure 2: A block diagram of the EHSS showing armband to BTE-device communication and the top view of the populated PCB.

2.5 Data Capture. Three male participants $(25-50 \mathrm{yrs}, 168-185 \mathrm{~cm}, 59-82 \mathrm{~kg}$ ) volunteered for a

10 human trial study designed to observe how energy harvesting trends change as the user transitions

11 between different environments and movement profiles and acquire to preliminary data for the design

12 of an integrated thermal energy harvesting garment. An outline of the human trial study is provided. 
1 First, a thermal imaging camera (FLIR E50) is used to map the distribution of the participants' skin

2 temperature. This information is used to compare energy harvesting areas on the body as well as define

3 an approximate skin temperature for each area. The participant then put on the headband or armband

4 and adjusted the compression of the band as they found comfortable. The subject then participated in

5 several pre-determined activities summarized in Table 1: walking, running, and resting. Each activity is

6 performed for at least $15 \mathrm{~min}$ while the data on the temperature, acceleration, humidity, and TEG

7 voltage was collected wirelessly on an Android tablet. The participant completed each activity in both an

8 indoor and outdoor setting.

9 Table 1: Thermal ranges for each activity and location during human trial studies.

\begin{tabular}{c|cc}
$\begin{array}{c}\text { Participant Activity } \\
\text { (15 min each) }\end{array}$ & $\begin{array}{c}\text { Indoor Ambient } \\
\text { Temperature }\left({ }^{\circ} \mathrm{C}\right)\end{array}$ & $\begin{array}{c}\text { Outdoor Ambient } \\
\text { Temperature }\left({ }^{\circ} \mathrm{C}\right)\end{array}$ \\
\hline Rest & $26 \pm 2$ & $30 \pm 2$ \\
Walk & $26 \pm 2$ & $28 \pm 3$ \\
Run & $26 \pm 2$ & $28 \pm 5$
\end{tabular}

10

\section{3. Results and Discussion}

123.1 In-Lab TEG Characterization. The results of laboratory-controlled TEG characterization tests,

13 presented in Fig. 3, provide two observations that are key to energy harvesting at low temperature

14 differentials across a TEG; first, convection over the TEG improves the steady state power generation

15 and second, heat sink properties are not proportional to power generation. The first trend in Fig. 3a

16 shows an increase in power generated when air convection is used to remove heat from the TEG (after

$1730 \mathrm{~min}$ of stagnant air). The second trend observed is that the power harvested is not proportional to

18 heat sink thermal properties. As shown, a decrease in power generated when the improved, $1.9^{\circ} \mathrm{C} / \mathrm{W}$

19 heat sink is used as compared to the $4.6{ }^{\circ} \mathrm{C} / \mathrm{W}$, both of which are improved compared to the $10{ }^{\circ} \mathrm{C} / \mathrm{W}$

20 heat sink. This is directly attributed excess cooling provided by the better heat sink, that without a 
change in heat input begins to limit the TEG performance. On a human, this result would be observed as a cooling sensation at the location of the TEG.

3

(1)
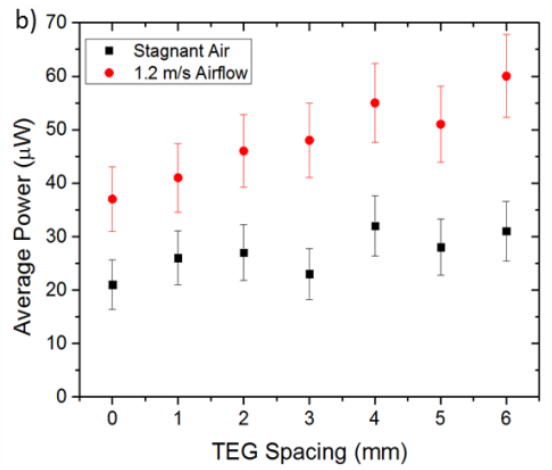

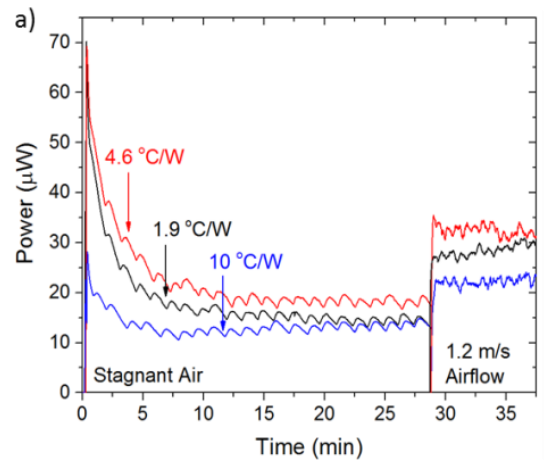

Figure 3: Heat sink comparison with constant heat input for a single TEG. (a) Air flow and spacing comparison showing the average power output of two TEGs with varied spacing and constant heat input. (b) The $4.6^{\circ} \mathrm{C} / \mathrm{W}$ heatsink displays the largest average power values. This heat sink is evaluated in both stagnant and moving air. (c) A comparison of the average power output between each of the heat sinks (units in ${ }^{\circ} \mathrm{C} / \mathrm{W}$ ) in constant airflow.

When the heat transfer from the heat sink is larger than what can be provided from skin, it may be possible that the cooling effect is observed at distances beyond the area of the skin that is directly in contact with the skin. In essence, this would induce a design constraint with respect to the spacing of two TEGs that exists at the heat spreading, skin side of the TEG. That is, when two TEGs are separated by a large distance, the total power harvested will be larger as compared to two TEGs intimately in contact.

It is important to note that this would suggest that the heat flow from the skin cannot be considered to be constant, as thermal effects would be observed from the neighboring TEG. To test this hypothesis, the effect of spacing between TEGs was evaluated by placing two TEGs in series at increasing spaces.

As shown in Fig. 3b, the total power harvested from the two TEGs increases with an increase in the spacing distance between TEGs. This trend is more pronounced with the addition of airflow across the 
device. A comparison of the spacing effects for each heat sink when subjected to air flow is provided in

2 Fig. 3c. As observed in the analysis of a single TEG (Fig. 3a), the $4.6^{\circ} \mathrm{C} / \mathrm{W}$ heat sink harvests more power

3

4

5

6

7

8

9

11

12

13

14

15

16

17

18

19 when compared to the 1.9 and $10{ }^{\circ} \mathrm{C} / \mathrm{W}$ heat sinks. As the spacing is increased ( 4 to $6 \mathrm{~mm}$ ), the increase power harvested is less impacted by the spacing of the TEGs, indicating that the spacing of the TEGs is sufficient to not impede on the neighboring TEG's heat flow (or pull). The importance of this result cannot be underestimated when it comes to designing multiple TEGs within close proximity for on-body energy harvesting. It is noted that potential factors that influence this spacing impact include the internal TEG design (fill factor, junction spacing, leg height) as well as the heat spreader and heat sink. From a comfort level, the rigid TEG spacing will also impact that the flexibility of package and therefore influence the thermal contact resistance between the skin and TEG. While additional work needs to be conducted to relate all of these factors, for the purpose of this work the construction of the multiple TEG package will assume that $6 \mathrm{~mm}$ spacing is sufficient to maximize the potential energy harvesting.

3.2 Body-Worn Device Data Results. Thermal imaging was first conducted to determine the average initial skin temperature of the forehead $\left(38^{\circ} \mathrm{C}\right)$ and the upper arm $\left(36^{\circ} \mathrm{C}\right)$ while in standard room conditions of $\sim 21{ }^{\circ} \mathrm{C}$. The pressure exerted by the device on the body was measured with a force sensitive resistor but was below the range of sensitivity so it is noted as $<0.1 \mathrm{psi}$. The following figures and results demonstrate the analysis procedure used in this study; however, not all data from individual participants is provided.

As shown in Fig. 4, the movement of subject 1 strongly influences the amount of power harvested from the TEGs. The instantaneous power, temperature, and movement intensity are shown in the top of Fig. 4 with respect to three movement scenarios: running, walking, and sitting. The temperature and power relationship is most obvious in Fig. 4a, outdoor running. However, the impact of ambient temperature 
on instantaneous power increases as the subject's movement decreases, indicating that the

2 instantaneous power is more influenced by air flow rather than dry-bulb temperature. When the subject

3 is seated, instantaneous power levels decrease to a negligible amount. The rate of energy harvesting

4 with each movement scenario is shown in Fig. 4d. The rate of energy harvesting while running is

5 approximately $0.021 \mathrm{~mJ} / \mathrm{s}$ which decreases to $0.013 \mathrm{~mJ} / \mathrm{s}$ while walking. The rate decreases to 0.001

$6 \mathrm{~mJ} / \mathrm{s}$ while the subject is seated. This vast difference in energy harvesting rates is another example of

7 the significant effect air flow has on TEG performance.

8
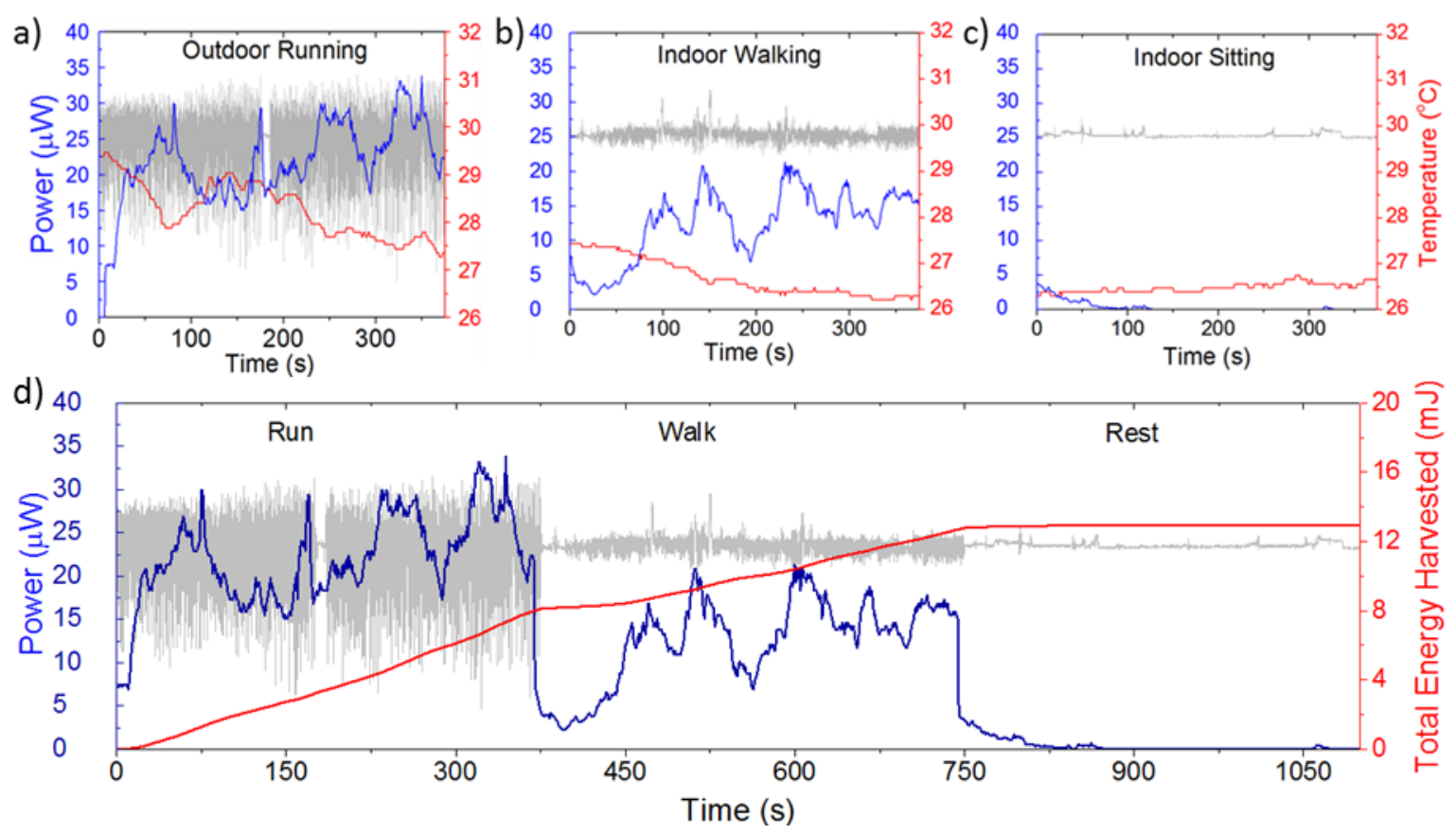

Figure 4: The instantaneous power (blue) from a five TEG armband as compared to ambient temperature (red) and accelerometer magnitude (gray) for the subject running (a) walking (b) and sitting (c). All scenarios compared only to movement displayed in (d) with accelerometer magnitude (gray) and instantaneous power (blue) and the rate of energy harvesting or additive amount of energy harvested per unit time (red). 
1 In summary, the energy harvesting data shows an increase in the energy harvesting rate with an

2 increase in movement and also confirms the inverse relationship of temperature and instantaneous

3 power. Based on the energy harvesting rates during various test scenarios, movement, or wind flow, is

4 considered a significant factor and changes in ambient temperature has less of an effect while the

5 subjects are running or walking. While seated, temperature became the dominant variable for

6 determining the rate of energy harvesting.

$7 \quad 3.3$ Temperature Analysis. A more thorough analysis was performed on a single subject to identify any

8 trends that may exist within the raw data. Instantaneous power, external temperature, and external

9 humidity plots for the running activity from the headband and armband are shown in Fig. 5,

10 respectively. An inverse correlation is observed between the ambient temperature and the

11 instantaneous power levels of the armband. The relative humidity does not appear to have a significant

12 effect on the instantaneous power generation during the running activity (Fig. 5a), however a slight

13 inverse correlation between humidity and energy harvesting is seen in the walking activity (Fig. 5b). As

14 air velocity across the heat sink increases (i.e. as the subject moves faster), it can be assumed that the

15 environmental microclimate surrounding the TEG can change in a short time period. Therefore, when

16 determining the temperature gradient across a wearable TEG, it must take into account both the

17 relative humidity and the temperature of the environment. This can be done with the use of a

18 psychometric chart or through the conversion of the measured dry-bulb temperature to wet-bulb

19 temperature via the method outlined in Stull. [30] 

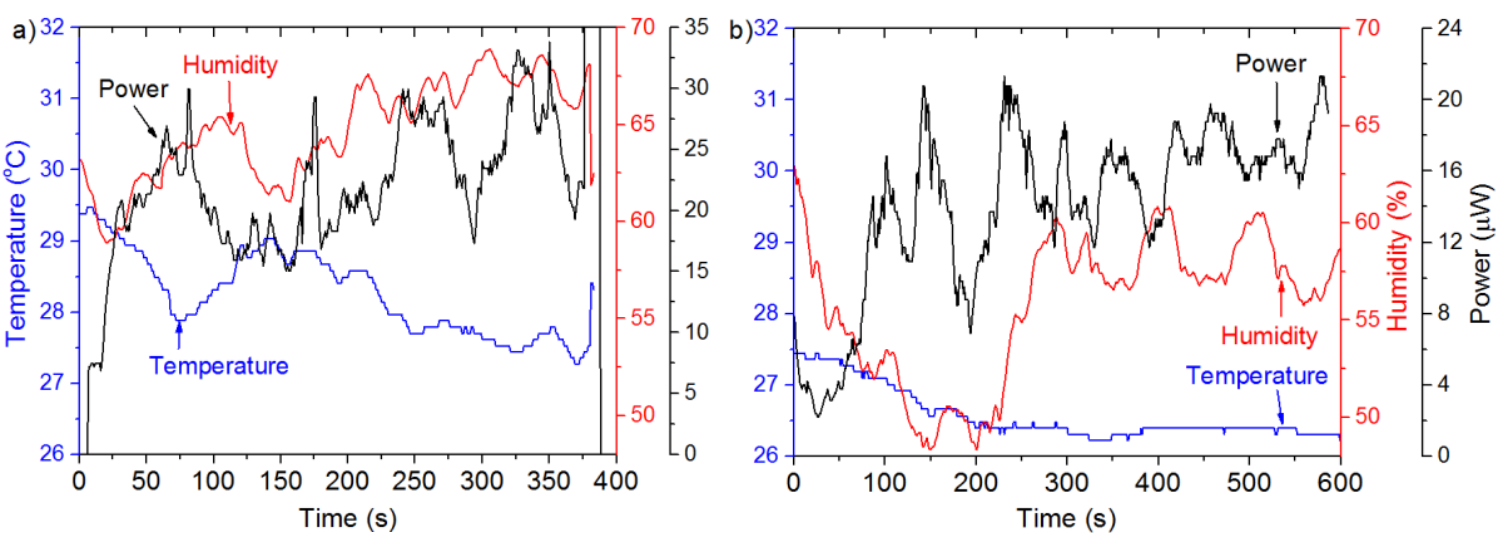

Figure 5: Comparison of power (black) harvested to that of temperature (blue) and of humidity (red) for the subject running (a) and walking (b).

The power and temperature correlation is examined in Fig. 6, showing two distinct clusters identified which correspond to each movement, walking and running. The clusters show a linear trend as seen in the armband output (Fig. 6a). A linear fit of the clusters results in an R-squared value greater than 0.5 for each cluster. While this confirms that the linear response is accurate, the large deviation from the linear trend from subject 2 indicates that the armband did not have secure contact with the skin and may have motion artifacts.

The relative humidity did not show a significant influence on the TEG power when compared directly in Fig. 5; however, when used to calculate the ambient wet-bulb temperature, a change in the shape of the clusters is noted. The linear trends seen in the dry-bulb temperature comparison from subject 2 is not visually apparent in Fig. 6b. This implies an even greater influence of movement on power output when one accounts for relative humidity. 
1

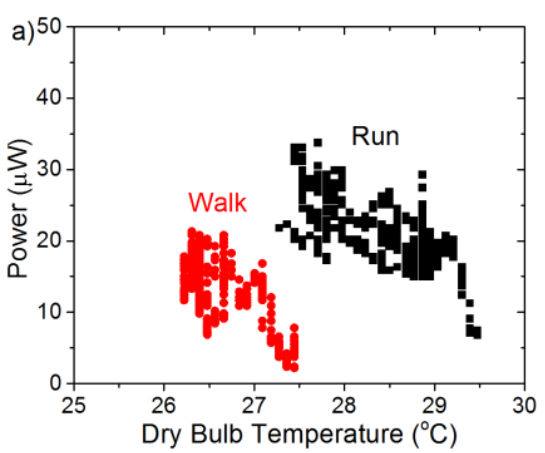

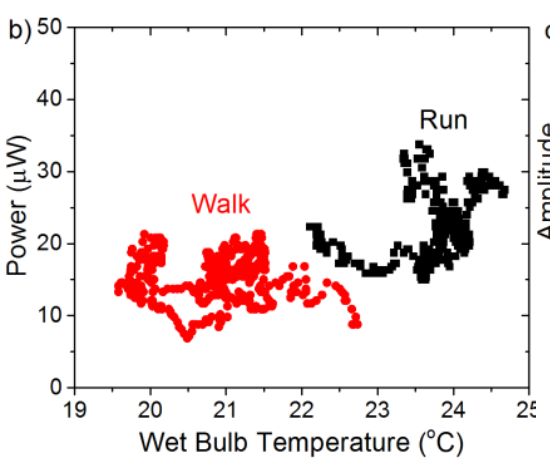

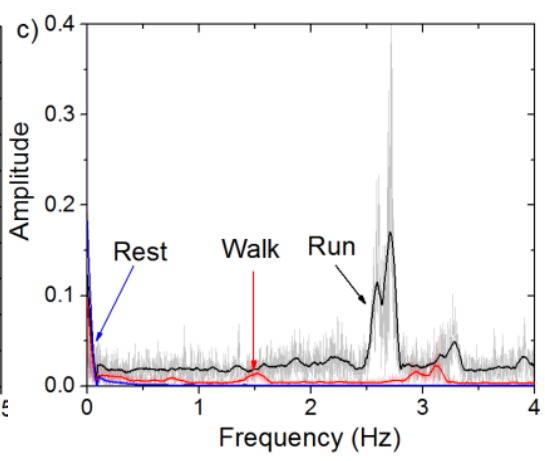

Figure 6: The instantaneous power clusters generated as compared to dry-bulb temperatures where

3 clusters are linear (a) and wet-bulb temperature where the clusters show more scattering (b) smoothed

$4 \quad$ FFT evaluation of accelerometer data from human trials comparing peak amplitude, intensity of foot

impact, to frequency pace of foot impact, for the armband (c)

To investigate the geometrical change in clusters with relative humidity considered, cluster area was measured with respect to each activity and subject. Namely, the horizontal and vertical ranges of each cluster were identified and the percent change in area between the dry-bulb temperature clusters and the wet-bulb temperature clusters was calculated as seen in the table below. the amount of power produced does not change.

Height Dry-Bulb Width Wet-Bulb Width Change in Area (\%)

\begin{tabular}{r|rr} 
Run & 18.78 & 2.20 \\
Walk & 14.55 & 1.22
\end{tabular}

12 
1 For the subject, the addition of humidity in the power and temperature comparison expanded the range

2 of temperatures recorded, resulting in an overall increase of the cluster area. The linearity of all data

3 decreased when wet-bulb temperature is considered.

4 This indicates that when estimating the power output of a body-worn thermal energy harvesting device,

5 one can increase the accuracy of the power estimate by using wet-bulb temperature regardless of

6 whether the subject is indoors or outdoors. To improve the accuracy of the estimate further, the

$7 \quad$ intensity of activity of the subject should be considered as well.

83.4 Accelerometer Analysis. The speed of someone as they move forward is correlated to the

9 frequency at which their foot strikes the ground when walking or running. Walking and running

10 repetitive movements with clear peak frequencies (Fig. 6c) while resting shows no clear movement

11 trends from the accelerometer data. The frequency of foot strike and intensity of foot strike (the

12 amplitude) were found by performing a Fast Fourier Transform (FFT) on the accelerometer data, seen in

13 the background of Fig. 6c. The data was smoothed using the Savitzky-Golay method with a 7 point

14 window (Fig. 6c foreground). The average frequency of foot strike when walking is between $1.5 \mathrm{and} 2 \mathrm{~Hz}$

15 with a mean amplitude of $0.055 \pm 0.01$. When running, the strike rate increases to $\sim 2.5 \mathrm{~Hz}$ with an

16 average impact amplitude of $0.311 \pm 0.05$. The frequency and intensity of the foot strikes increase with

17 the subjects' pace.

18 Similar to the rate of energy harvesting, the strike intensity nearly doubles as the participant speeds up

19 from a walk to a run, confirming the close correlation of thermal energy harvesting efficiency to

20 movement. When performing the analysis, only the values from the $\mathrm{x}$-axis of the accelerometer were

21 evaluated. The data from each axis was compared and found to have little to no trend variation from

22 that of the $\mathrm{x}$-axis data. 
13.5 Subject to Subject Variability The accelerometer data was converted to velocity for further

2 analysis. To do so, the raw data was converted from units of $g$ (force) to $\mathrm{m} / \mathrm{s}^{2}$ and then integrated. The

3 following figures investigate the reliance of thermal power harvested to subject velocity and to wet-bulb

4 temperature. Fig. 7a and $7 \mathrm{~b}$ compare power trends while each subject was running wearing the

5 armband. The data confirms larger amounts of power at higher velocities, but the contour plots

6 between subjects are vastly different. One reason for this is the level of contact the armband makes

7 with the skin for each subject. It is difficult to quantify exact contact pressure due to differing comfort

8 levels and amounts of hair between subjects. As the contact pressure changes, the area of the contour is

9 likely to change as well. In the same manner, the use of different TEGs, heat spreaders, or heat sinks will

10 affect the size and layout of the plot. 

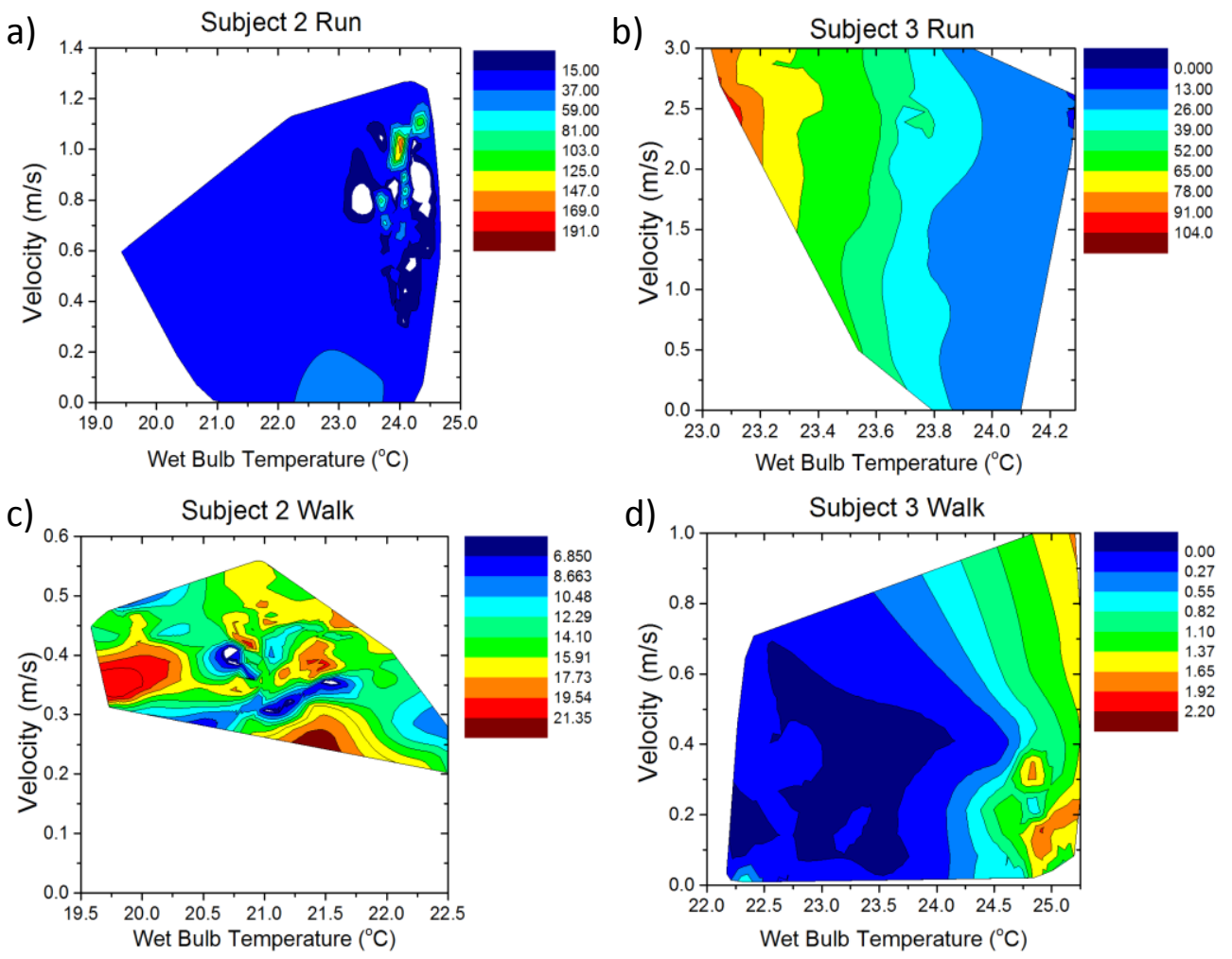

Figure 7: Contour mapping of power generated in $\mu \mathrm{W}$ for a color scale from blue to red (red is the largest power value) with respect to wet-bulb temperature and subject velocity. The activity from one subject is shown on the left and another is shown on the right.

A similar trend between the power response to body location of the wearable device can be noted when the subject is walking, as seen in Fig. $7 c$ and $7 d$. Again, the areas with largest power generation are associated with lower temperatures and higher velocities. Discrepancies between the power generation trends are due to subject variability. To see if these observations apply when all movements and subjects are directly compared to each other, each activity from each subject was mapped on the contour plot seen in Fig. 8. This confirms the trends previously noted: the most thermal power will be

11 generated when the temperature gradient is largest and when the subject is generating the most air 12 flow over the cold side of the TEGs. It should be noted that Fig. 7 and Fig. 8 allow for the prediction of 13 the energy harvested amongst individuals and groups of individuals. This prediction allows for the 
1 boundaries that support power management profiles for future self-powered devices. In addition, the

2 methods serve as a benchmark for materials and device improvement.

3 When comparing the on body location of the TEGs, one must look at the use case scenario. While the

4 armband has lower instantaneous power levels, the rate of energy harvesting while the subject is

5 moving is significantly higher than the headband; indicating that it would harvest more energy over a

6 longer period of time while in motion. This suggests that the armband platform is better suited for a

7 consumer who is consistently active throughout the day. In future scenarios, power generation trends

8 will also depend on the location of TEGs on the body. Each potential location on the body has benefits

9 and drawbacks in terms of ideal location for thermal energy harvesting. Therefore, it is necessary to

10 consider a consumer who may have different movement scenarios throughout the day. The results of

11 the thermal energy harvesting body map guide the design and placement of other wearable energy

12 harvesting devices to meet this requirement.

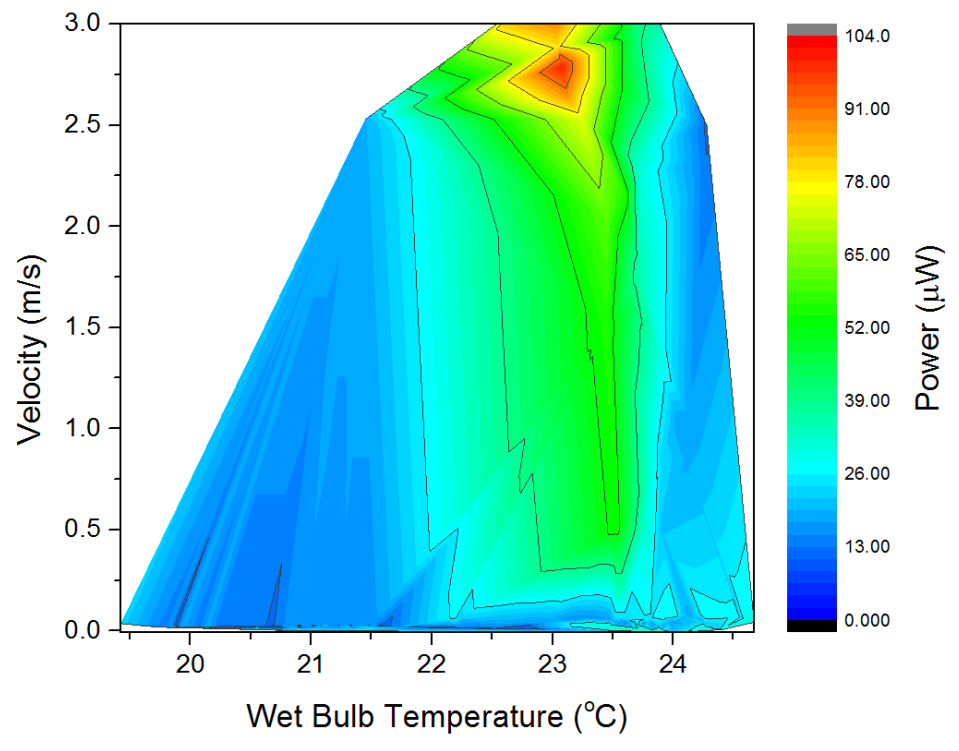

Figure 8: Contour map of thermal energy harvested from the armband as a function of subject speed

15 and wet-bulb temperature for all subjects. On the scale from blue to red, blue represents the lowest instantaneous power value in $\mu \mathrm{W}$. 
In summary, the work completed in this study determine the effect environmental and human factors have on thermal energy generator (TEG) performance in a variety of scenarios. A test method for assessing the performance of a TEG in a wearable form is detailed and demonstrated. The fabrication

5 procedure of an energy harvesting device demonstrates a method of integrating rigid devices into a flexible substrate. The wearable device is used in a human trial which covered a series of activities in different environmental conditions. The results of these trials demonstrate the significant effect of movement, or convection, on thermal energy harvesting. Humidity levels do not have a significant correlation to power; however wet-bulb temperature must be taken into consideration due to the additional cooling effect of evaporation on temperature. While the amount of power generated varied between individuals, the combined data sets match expected trends. The combined data quantifies how

12 a wearable device will perform throughout daily activities for a group of individuals. This allows someone to define the operating scenario for a self-powered wearable device while choosing the most appropriate design for a particular application. It also sets a benchmark for exploring how textiles can enable the design of unique wearable devices which will lead to further investigation into quantifying the effect that the construction of a textile has on TEG performance as well as on consumer comfort.

\section{Acknowledgements}

This work is supported by the US National Science Foundation through Nanosystems Engineering

Research Center for Advanced Self-Powered Systems for Integrated Sensors and Technologies under Grant EEC 1160483.

\section{References}

22 [1] K. Parsons, Introduction to thermal comfort standards, in: Conf. Mov. Therm. Comf. Stand., 2001: pp. 19-30. http://nceub.org.uk/uploads/Paper03_Parsons.pdf. 
1 [2] Y. Li, The Science of Clothing Comfort, Text. Prog. 31 (2010) 1-135.

2

3 [3] P.O. Fanger, Assessment of thermal comfort practice, Occup. Environ. Med. 30 (1973) 313-324.

4

5

6

7

8

9

10

11

12 doi:10.1080/00405160108688951. doi:10.1136/oem.30.4.313.

[4] R. De Dear, G. Brager, U.C. Berkeley, Developing an adaptive model of thermal comfort and preference, ASHRAE Trans. 104 (1998) 1-18. https://escholarship.org/uc/item/4qq2p9c6.pdf\%5Cnhttp://escholarship.org/uc/item/4qq2p9c6. pdf\%5Cnhttp://repositories.cdlib.org/cedr/cbe/ieq/deDear1998_ThermComPref.

[5] C. Edifici, C. Codrea, M. Pollak, P. Spies, Human Body Energy Harvesting Thermogenerator for Sensing Applications, in: Int. Conf. Sens. Technol. Appl., 2007: pp. 366-372. doi:10.1109/SENSORCOMM.2007.42.

[6] C.R. Saha, T. O’Donnell, N. Wang, P. McCloskey, Electromagnetic generator for harvesting energy from human motion, Sensors Actuators, A Phys. 147 (2008) 248-253. doi:10.1016/j.sna.2008.03.008.

[7] A. Potnuru, Y. Tadesse, Characterization of Pyroelectric Materials for Energy Harvesting from Human Body, Integr. Ferroelectr. 150 (2014) 23-50. doi:10.1080/10584587.2014.873319.

[8] F. Suarez, A. Nozariasbmarz, D. Vashaee, M.C. Ozturk, Designing Thermoelectric Generators for Self-Powered Wearable Electronics, Energy Environ. Sci. (2016) 1-19. doi:10.1039/C6EE00456C.

[9] A. Gagge, R. Gonzalez, Mechanisms of Heat Exchange: Biophysics and Physiology, Handb. Physiol. Environ. Physiol. (1996) 45-84. doi:10.1002/cphy.cp040104.

[10] K. Parsons, Human Thermal physiology and thermoregulation, in: Hum. Therm. Environ., 2014: pp. 31-48. 
1 [11] A. Gagge, Y. Nishi, Heat exchange between human skin surface and thermal environment, in: Handb. Physiol. - React. to Environ. Agents, 1977: pp. 69-92. doi:10.1002/cphy.cp090105.

[12] Z. Wang, V. Leonov, P. Fiorini, C. Van Hoof, Realization of a wearable miniaturized thermoelectric generator for human body applications, Sensors Actuators A Phys. 156 (2009) 95-102. doi:10.1016/j.sna.2009.02.028.

[13] J.W. Stevens, Optimal design of small $\Delta T$ thermoelectric generation systems, Energy Convers. Manag. 42 (2001) 709-720. doi:10.1016/\$0196-8904(00)00099-6.

[14] S. Jo, M. Kim, M. Kim, H. Kim, Y. Kim, Human Body Heat Energy Harvesting Using Flexible Thermoelectric Generator for Autonomous Microsystems, Rsc.Org. (2012) 839-841. http://www.rsc.org/images/loc/2012/pdf/M.9.194.pdf.

[15] V. Leonov, C. Van Hoof, R.J.M. Vullers, Thermoelectric and Hybrid Generators in Wearable Devices and Clothes, in: 2009 Sixth Int. Work. Wearable Implant. Body Sens. Networks, IEEE, 2009: pp. 195-200. doi:10.1109/BSN.2009.10.

[16] A. Yadav, K.P. Pipe, M. Shtein, Fiber-based flexible thermoelectric power generator, J. Power Sources. 175 (2008) 909-913. doi:10.1016/j.jpowsour.2007.09.096.

[17] V. Leonov, Energy Harvesting for Self-Powered Wearable Devices, in: A. Bonfiglio, D. De Rossi (Eds.), Wearable Monit. Syst., Springer US, Boston, MA, 2011: pp. 27-49. doi:10.1007/978-14419-7384-9.

[18] V. Leonov, R.J.M. Vullers, Wearable electronics self-powered by using human body heat: The state of the art and the perspective, J. Renew. Sustain. Energy. 1 (2009) 62701. doi:10.1063/1.3255465.

[19] K.T. Settaluri, H. Lo, R.J. Ram, Thin Thermoelectric Generator System for Body Energy Harvesting, J. Electron. Mater. 41 (2011) 984-988. doi:10.1007/s11664-011-1834-3. 
1 [20] J.-H. Bahk, H. Fang, K. Yazawa, A. Shakouri, Flexible thermoelectric materials and device

optimization for wearable energy harvesting, J. Mater. Chem. C. 3 (2015) 10362-10374. doi:10.1039/c5tc01644d.

[21] A. Majumdar, S. Mukhopadhyay, R. Yadav, Thermal properties of knitted fabrics made from cotton and regenerated bamboo cellulosic fibres, Int. J. Therm. Sci. 49 (2010) 2042-2048. doi:10.1016/j.ijthermalsci.2010.05.017.

[22] Z. Lu, H. Zhang, C. Mao, C.M. Li, Silk fabric-based wearable thermoelectric generator for energy harvesting from the human body, Appl. Energy. 164 (2016) 57-63. doi:10.1016/j.apenergy.2015.11.038.

[23] V. Leonov, R.J.M. Vullers, Wearable Thermoelectric Generators for Body-Powered Devices, J. Electron. Mater. 38 (2009) 1491-1498. doi:10.1007/s11664-008-0638-6.

[24] J.E. Mark, Polymer Data Handbook, 2nd ed., Oxford University Press, 2009. http://app.knovel.com/web/toc.v/cid:kpPDHE0004 (accessed November 18, 2015).

[25] R.C. Webb, A.P. Bonifas, A. Behnaz, Y. Zhang, K.J. Yu, H. Cheng, M. Shi, Z. Bian, Z. Liu, Y.-S. Kim, W.-H. Yeo, J.S. Park, J. Song, Y. Li, Y. Huang, A.M. Gorbach, J. Rogers, Ultrathin conformal devices for precise and continuous thermal characterization of human skin., Nat. Mater. 12 (2013) 93844. doi:10.1038/nmat3755.

[26] A. Martinez-Nicolas, E. Ortiz-Tudela, M.A. Rol, J.A. Madrid, Uncovering different masking factors on wrist skin temperature rhythm in free-living subjects., PLoS One. 8 (2013) e61142. doi:10.1371/journal.pone.0061142.

[27] P.D. Mitcheson, E.M. Yeatman, G.K. Rao, A.S. Holmes, T.C. Green, Energy Harvesting From Human and Machine Motion forWireless Electronic Devices, Proc. IEEE. 96 (2008) 1457-1486. doi:10.1109/JPROC.2008.927494. 
1 [28] M. Lossec, B. Multon, H. Ben Ahmed, C. Goupil, Thermoelectric generator placed on the human body: system modeling and energy conversion improvements, Eur. Phys. J. Appl. Phys. 52 (2010) 11103. doi:10.1051/epjap/2010121.

4
[29] M. Guan, K. Wang, D. Xu, W.-H. Liao, Design and experimental investigation of a low-voltage thermoelectric energy harvesting system for wireless sensor nodes, Energy Convers. Manag. 138 (2017) 30-37. doi:http://dx.doi.org/10.1016/j.enconman.2017.01.049.

[30] R. Stull, Wet-Bulb Temperature from Relative Humidity and Air Temperature, J. Appl. Meteorol. Climatol. 50 (2011) 2267-2269. doi:10.1175/JAMC-D-11-0143.1. 